\title{
Tattoo Koebnerization and Severe Pulmonary Hypertension
}

\author{
Matthew Patel and Karim Ladak, MD
}

A n African-American man, aged 35 years, was admitted to the hospital with progressively worsening dyspnea of 1.5 years duration. Pre-morbid function was excellent. History and examination were unremarkable for other features of respiratory, cardiac and connective tissue disease. He had tattoos that were 10 years of age, but denied travel, substance use, exposures, and sick contacts. Family history was non-contributory. Past medical history included hypertension, idiopathic uveitis, and a recent JAK2-negative polycythemia. His only medications were steroid eye-drops.

Admission pulse oximetry was $87 \%$. He was afebrile and normotensive. Chest radiography revealed enlarged pulmonary arteries but otherwise normal lung fields. Outpatient computed tomography (CT) of the chest done 4 months prior showed similar findings. Echocardiogram demonstrated right heart enlargement, but normal left heart (Figure 1). Right heart catheterization showed severe pulmonary arterial hypertension with pressures of $108 / 55 \mathrm{mmHg}$, yet normal wedge pressures. Workup for secondary causes of hypertension was positive for antinuclear antibodies (ANA 1:640), while all other testing for features of autoimmune disease was normal.

During hospitalization, a sliver of one of his tattoos became raised and urticarial. Skin biopsy demonstrated an extensive epithelioid, non-caseating granulomatous infiltrate associated with exogenous black carbon pigment surrounded by small lymphocytes (Figure 2). Special stains were negative. This tattoo Koebnerization was consistent with sarcoidosis. Repeat chest CT displayed new findings of subtle, central right upper lobe fibrosis with peribronchiolar honeycombing and tiny scattered perivascular lymphadenopathy, without hilar lymphadenopathy. The patient started vasodilators and glucocorticoids with rapid improvement and was discharged on ambrisentan, tadalafil, and methylprednisolone. Adalimumab was started as a steroid-sparing agent.

\section{Discussion}

Sarcoidosis is a multisystem disease of unknown etiology characterized by epithelioid non-caseating granulomas with usual onset between 20 years and 40 years of age and is more commonly seen in African Americans (as in this patient) relative to Caucasians. ${ }^{1}$ Antigenic triggers are unknown, but tattoos have been proposed as potential antigenic stimuli for granuloma formation. ${ }^{2,3}$ Sarcoidal skin reactions in tattoos are rare, but reports date back to $1952 .{ }^{4}$ These Koebnerization responses primarily affect red (cinnabar), black (ferric oxide), and blue-black tattoos, and often precede other systemic disease manifestations. ${ }^{5}$ In the diagnosis of sarcoidosis, tattoos serve as a safe and convenient site for biopsy.

Sarcoidosis-associated pulmonary hypertension (SAPH) is an increasingly recognized cause of morbidity and mortality whose prevalence ranges from $5 \%$ to $20 \%{ }^{6}$ It is categorized
Corresponding Author: Matthew Patel, 34 Southlea Avenue, Toronto, Ontario M4G 3L9 Canada, Email: matthewpatel@rcsi.com
Received: October 22, 2018

Revised: May 25, 2019

Accepted: June 24, 2019

doi: $10.3121 / \mathrm{cmr} .2019 .1469$

Keywords: Sarcoidosis; Pulmonary hypertension; Koebnerization; Tattoo reaction 


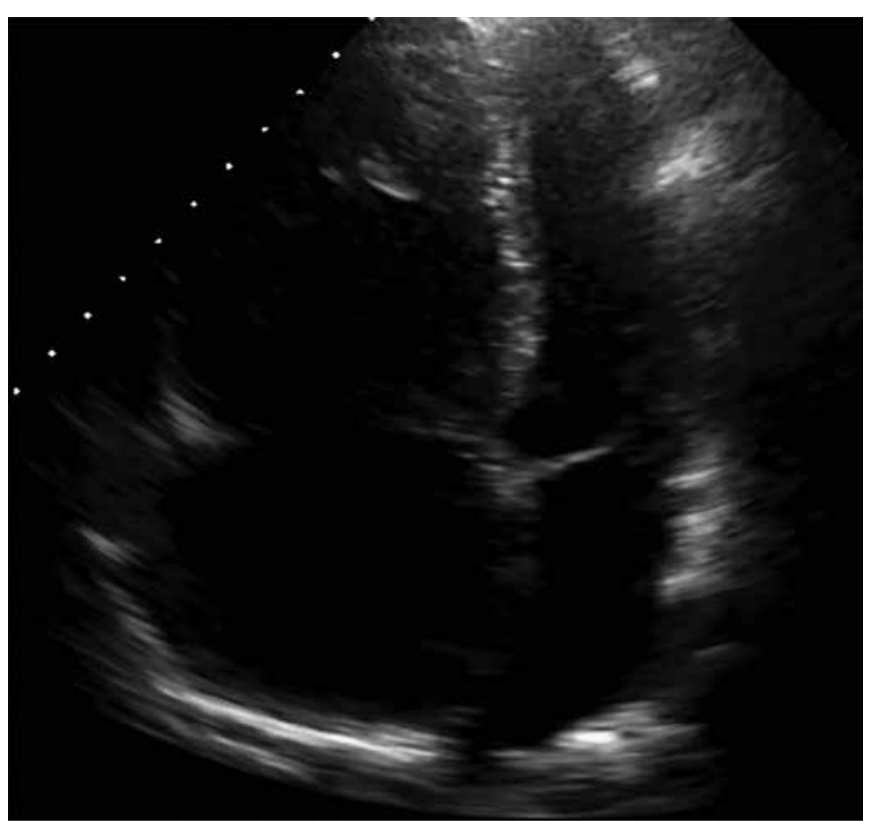

Figure 1. Echocardiogram in four-chamber view. Grossly enlarged right ventricle (top left) and right atrium (bottom left), with bowing of the interventricular septum into the left ventricle.

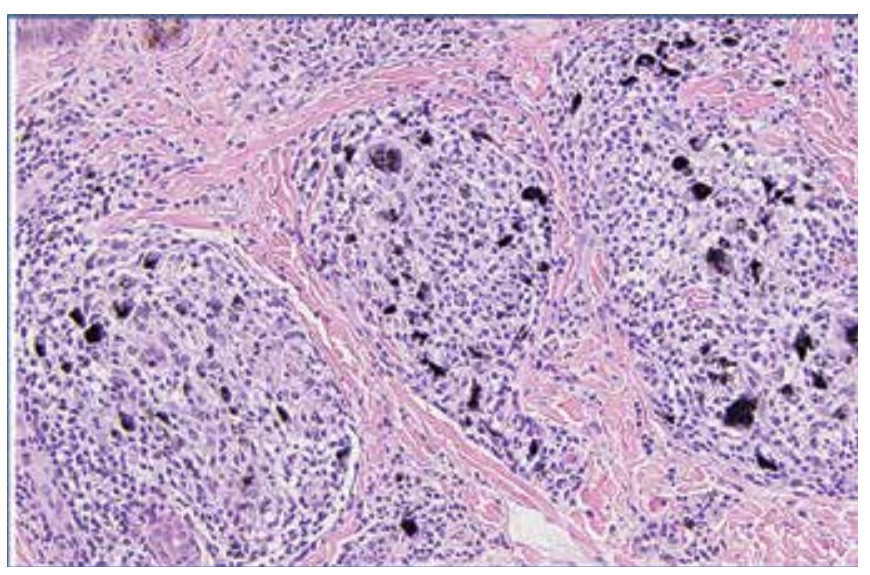

Figure 2. Extensive epithelioid, non-caseating granulomatous infiltrate associated with exogenous black carbon pigment, surrounded by small lymphocytes.

as WHO class 5 pulmonary hypertension, occurring through various mechanisms including intrinsic vasculopathy, mechanical obstruction of vasculature by lymphadenopathy, fibrotic destruction of vascular beds, and hypoxic vasoconstriction. ${ }^{7}$ SAPH usually occurs in radiographically advanced cases, but vasculopathic SAPH does rarely present in radiologic stage 0 , as in our patient. ${ }^{6} \mathrm{SAPH}$ should be suspected when dyspnea exceeds the degree of radiographic abnormalities, and if respiratory symptoms are resistant to anti-inflammatory therapy. Echocardiography and 6-minute walk test are reasonable screening tools; however, right heart catheterization is the gold standard for diagnosis. ${ }^{6}$ Unfortunately, the prognosis is grave, with a 5 -year mortality of $45 \%{ }^{8}$
While the patient's ethnicity, uveitis, interstitial lung disease, and skin biopsy findings support the diagnosis of SAPH, the degree of chest CT findings are not as extensive as one may expect, and the positive ANA is unusual. While a firm diagnosis of pulmonary sarcoidosis cannot be made without a lung/lymph node biopsy, extensive clinical and serologic evaluation for connective tissue disease was negative, and a lack of pulmonary interstitial findings does not exclude $\mathrm{SAPH} .{ }^{5}$

\section{References}

1. Newman LS, Rose CS, Maier LA. Sarcoidosis. N Engl J Med. 1997;336(17):1224-1234.

2. Agostini C, Adami F, Semenzato G. New pathogenetic insights into the sarcoid granuloma. Curr Opin Rheumatol. 2000;12(1):71-76.

3. Papageorgiou PP, Hongcharu W, Chu AC. Systemic sarcoidosis presenting with multiple tattoo granulomas and an extratattoo cutaneous granuloma. J Eur Acad Dermatol Venereol. 1999;12(1):51-53.

4. Lubeck G, Epstein E. Complications of tattooing. Calif Med. 1952;76(2):83-85

5. O'Beirne SL, O'Dwyer DN, Walsh SM, Dodd JD, Crotty TB, Donnelly SC. The lady with the dragon tattoo. Ir J Med Sci. 2017;186(1):157-160.

6. Shlobin O, Baughman R. Sarcoidosis-Associated Pulmonary Hypertension. Semin Respir Crit Care Med. 2017;38(04):450-462.

7. Diaz-Guzman E, Farver C, Parambil J, Culver DA. Pulmonary hypertension caused by sarcoidosis. Clin Chest Med. 2008;29(3):549-563.

8. Boucly A, Cottin V, Nunes H, et al. Management and long-term outcomes of sarcoidosis-associated pulmonary hypertension. Eur Respir J. 2017;50(4):1700465.

\section{Author Affiliations}

Matthew Patel*, and Karim Ladak, MD广

*Medical Student, Royal College of Surgeons in Ireland, Dublin, Ireland

†Department of Rheumatology, Hospital for Special Surgery, Cornell University, New York City, USA. Current affiliation: Department of Rheumatology, McMaster University, Hamilton, Ontario, Canada. 